ZU-TH 14/07

\title{
Radiative corrections to bino-stop co-annihilation
}

\author{
A. Freitas \\ Institut für Theoretische Physik, Universität Zürich, \\ Winterthurerstrasse 190, CH-8057 Zürich, Switzerland
}

\begin{abstract}
In co-annihilation scenarios, the weakly interacting dark matter particle (WIMP) is close in mass to another particle that can decay into the WIMP. As a result, the other particle does not freeze out before the WIMP in the early universe, and both contribute to the effective dark matter annihilation cross-section. Since the heavier particle does not need to be weakly interacting, the co-annihilation processes are in general subject to sizeable radiative corrections. Here this is analyzed for the example of neutralino-stop co-annihilation in supersymmetry. The leading QCD corrections are calculated and it is found that they have a large effect on the effective annihilation cross-section, reaching more than $50 \%$ in some regions of parameter space.
\end{abstract}


In recent years, the existence of dark matter in the universe has been firmly established by various different astrophysical experimental methods. From the cosmic microwave background and large scale structure, the dark matter density is determined to be $\Omega_{\mathrm{CDM}} h^{2}=$ $0.1106_{-0.0075}^{+0.0056}$ [1], where $\Omega_{\mathrm{CDM}}$ is the ratio of the dark matter energy density to the critical density $\rho_{c}=2 H_{0}^{2} /\left(8 \pi G_{\mathrm{N}}\right)$ with the Hubble constant $H_{0}=h \times 100 \mathrm{~km} / \mathrm{s} / \mathrm{Mpc}$ and Newton's constant $G_{\mathrm{N}}$. A promising explanation for the nature of dark matter in agreement with direct observations and simulations of galaxy formation are weakly interacting massive particles (WIMPs). While the Standard Model does not encompass an appropriate particle for this purpose, a suitable candidate can arise from many extensions of the Standard Model, such as supersymmetry, extended gauge groups or non-anomalous global symmetry groups.

Due to the requirement that the dark matter particle is weakly interacting, its annihilation cross-section typically receives only small radiative corrections of $\mathcal{O}(\%) 1$ This allows to make robust predictions of the present dark matter density within a certain model for a given choice of parameters. Assuming the standard cosmological model, the evolution of the dark matter density from the time of freeze-out from thermal equilibrium until the present time can be computed based only on the Boltzmann equation and the thermally averaged annihilation cross-section (see for example Ref. [3]).

Besides predicting a dark matter candidate, most of the Standard Model extensions also introduce a large spectrum of additional particles. In supersymmetry, for instance, every Standard Model particle has a supersymmetric partner. If the mass of one of these particles is close to the WIMP mass, it would not yet be decoupled from thermal equilibrium at the freeze-out temperature of the WIMP and thus influences the WIMP annihilation. This mechanism is called co-annihilation and effectively lowers the present day relic density [4]. It is interesting to note that the co-annihilating particle can have very different quantum numbers than the WIMP, as long as it decays into a final state which includes the WIMP. In particular, the co-annihilating particle does not need to be weakly interacting. In some supersymmetric scenarios, co-annihilation occurs between the lightest neutralino (as the WIMP) and the stau or stop, which participate in electromagnetic, and in the latter case also strong, interactions. As a result, radiative corrections can be very important in coannihilation processes.

In this letter, QCD corrections to co-annihilation processes are analyzed. For concreteness, the specific process of neutralino-stop co-annihilation within the Minimal Supersymmetric Standard Model (MSSM) is considered. In many supersymmetry breaking scenarios, the light stop $\tilde{t}_{1}$ is predicted to be the lightest squark state due to large running and mixing effects, so that it is not improbable that the $\tilde{t}_{1}$ can get close in mass to the lightest neutralino $\tilde{\chi}_{1}^{0}$. This scenario is in particular motivated by electroweak baryogenesis, see e.g. Ref. [5]. If the mass difference $m_{\tilde{t}_{1}}-m_{\tilde{\chi}_{1}^{0}}$ is small, and all other superpartners are significantly heavier than the $\tilde{t}_{1}$, there are effectively three contributing processes to the evolution of the dark matter density,

$$
\tilde{\chi}_{1}^{0} \tilde{\chi}_{1}^{0} \rightarrow X, \quad \tilde{\chi}_{1}^{0} \tilde{t}_{1} \rightarrow X, \quad \tilde{t}_{1} \tilde{t}_{1}^{(*)} \rightarrow X,
$$

\footnotetext{
${ }^{1}$ An exception are annihilation processes that are suppressed at tree-level, but this suppression is lifted at the loop level, see e.g. Ref [2].
} 
(a)
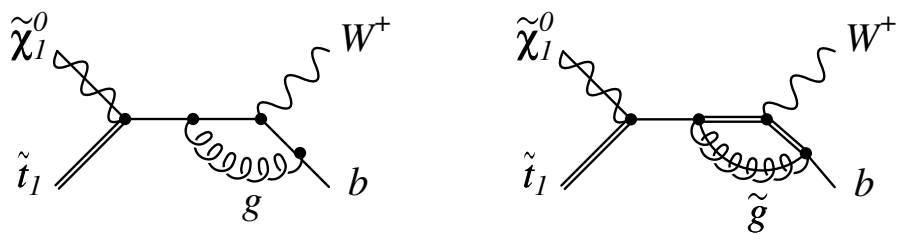

(b)
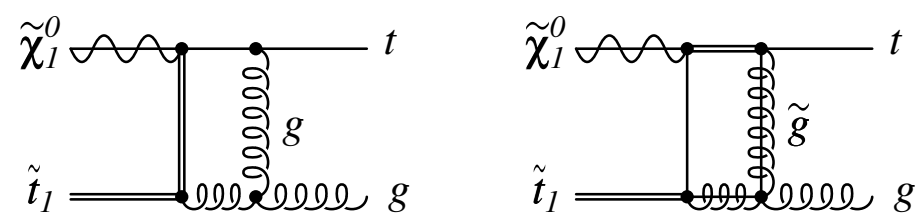

Figure 1: Some loop diagrams for the $\mathcal{O}\left(\alpha_{\mathrm{s}}\right)$ corrections to (a) $\tilde{\chi}_{1}^{0} \tilde{t}_{1} \rightarrow W^{+} b$ and (b) $\tilde{\chi}_{1}^{0} \tilde{t}_{1} \rightarrow t g$.

where $X$ stands for some Standard Model particles. The relic density within a stop-neutralino co-annihilation scenario has been first calculated in Ref. [6], using tree-level formulae for the these three annihilation cross-sections. For a typical parameter point with a predominantly bino $\tilde{\chi}_{1}^{0}$ of $118 \mathrm{GeV}$ and a predominantly R-chiral stop of $138 \mathrm{GeV}$, the dark matter density is predicted to be $\Omega h^{2}=0.112$, using DARKSUSY 4.1 [7]. Here $\tilde{\chi}_{1}^{0} \tilde{\chi}_{1}^{0}$ annihilation contributes only about $5 \%$ to the total averaged annihilation cross-section, while $\tilde{\chi}_{1}^{0} \tilde{t}_{1}$ and $\tilde{t}_{1} \tilde{t}_{1}^{*}$ annihilation contribute $85 \%$ and $10 \%$, respectively. In the following, QCD radiative corrections to the last two processes will be studied in detail.

$\tilde{\chi}_{1}^{0} \tilde{t}_{1}$ annihilation: The annihilation of $\tilde{\chi}_{1}^{0} \tilde{t}_{1}$ into Standard Model particles receives contributions from several channels. For the aforementioned parameter point with $\tilde{\chi}_{1}^{0} \sim \tilde{B}_{\text {, }}$ $m_{\tilde{\chi}_{1}^{0}}=118 \mathrm{GeV}$ and $\tilde{t}_{1} \sim \tilde{t}_{\mathrm{R}}, m_{\tilde{t}_{1}}=138 \mathrm{GeV}$, one finds

$$
\begin{array}{lr}
\tilde{\chi}_{1}^{0} \tilde{t}_{1} \rightarrow W^{+} b & 49.5 \% \text { contribution to the thermally averaged } \tilde{\chi}_{1}^{0} \tilde{t}_{1} \text { cross-section, } \\
\tilde{\chi}_{1}^{0} \tilde{t}_{1} \rightarrow t g & 47.5 \% \\
\tilde{\chi}_{1}^{0} \tilde{t}_{1} \rightarrow t \gamma & 1.0 \%, \\
\tilde{\chi}_{1}^{0} \tilde{t}_{1} \rightarrow t Z & 2.0 \%,
\end{array}
$$

while all other kinematically allowed final states are negligible. Since the first two processes are by far dominant, this work concentrates on the radiative corrections to $\tilde{\chi}_{1}^{0} \tilde{t}_{1} \rightarrow W^{+} b$ and $\tilde{\chi}_{1}^{0} \tilde{t}_{1} \rightarrow t g$. Typical diagrams for the $\mathcal{O}\left(\alpha_{\mathrm{s}}\right)$ virtual corrections are shown in Fig. 1. Since the tree-level processes include quark-squark-neutralino vertices, the QCD corrections with gluon exchange cannot be separated from the SUSY-QCD corrections with gluino exchange. Therefore the complete SUSY-QCD corrections of order $\mathcal{O}\left(\alpha_{\mathrm{s}}\right)$ will be considered.

The technical methods for the calculation are well established. The virtual loop diagrams have been generated with FEYNARTS 3.0 [8], while the algebraic reduction to standard matrix elements was performed with the help of FEYNCALC 2.2 [9]. In the loop integrals UV divergencies occur, which are canceled through the renormalization of mass, mixing and 


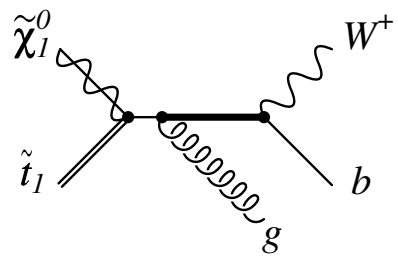

Figure 2: One diagram in the real gluon corrections to $\tilde{\chi}_{1}^{0} \tilde{t}_{1} \rightarrow W^{+} b$ where the intermediate top-quark propagator can become on-shell, indicated by a thick line.

coupling parameters. The masses have been renormalized according to the on-shell scheme, while for the strong coupling constant the $\overline{\mathrm{MS}}$ scheme with six-flavor SUSY-QCD running has been chosen. Instead of introducing a counterterm for the stop and sbottom mixing angles [10], the squark sector has been renormalized by using matrix-valued mass counterterms [11. The two approaches lead to different definitions of the renormalized mixing angles, but are equivalent (up to higher orders) when relating physical observables.

The loop diagrams with gluon exchange also lead to the IR divergencies, that have been regularized by a gluon mass. They need to be combined with the contributions with real gluon emission in order to arrive at an IR finite complete result. Here a word of caution is in order for the real corrections to the process $\tilde{\chi}_{1}^{0} \tilde{t}_{1} \rightarrow W^{+} b$. In the diagram shown in Fig. 2 , the top propagator that is shown as a thick line can be become on-shell. In this kinematical region, however, it would be a contribution to the process $\tilde{\chi}_{1}^{0} \tilde{t}_{1} \rightarrow t g$ with the subsequent decay $t \rightarrow W^{+} b$. Therefore the terms with a resonant top propagator need to be subtracted from the matrix element of $\tilde{\chi}_{1}^{0} \tilde{t}_{1} \rightarrow W^{+} b+g$. On the other hand, the interference terms between an amplitude with a resonant top propagator and a non-resonant amplitude are retained in this channel.

For the numerical evaluation, the loop integrals are computed with the package LooPTools [12, while the phase space for the real corrections is integrated numerically using Monte Carlo methods. The real radiation phase space has been mapped onto the integration variables in an optimized way so as to improve the result in the regions where the gluon(s) become(s) soft or collinear (the collinear case only occurs for the process $\tilde{\chi}_{1}^{0} \tilde{t}_{1} \rightarrow t g+g$ ). After combining virtual and real corrections, it has been checked that the soft and collinear singularities drop out of the total result, so that it does not depend on the gluon mass regulator within phase space integration errors.

In order to simplify the presentation of the numerical results, one can observe that the dynamics of co-annihilation are mainly governed by the particle masses, whereas mixing effects play a minor role. Thus for simplicity, here it is assumed that the lightest neutralino $\tilde{\chi}_{1}^{0}$ is a pure bino $\tilde{B}$, while the light stop $\tilde{t}_{1}$ is purely the partner of the right-handed stop 2 . The size of the SUSY-QCD corrections in this scenario is illustrated in Fig. 3, As can be seen from the figure, the corrections to $\tilde{\chi}_{1}^{0} \tilde{t}_{1} \rightarrow W^{+} b$ amount to only a few per-cent, while the process $\tilde{\chi}_{1}^{0} \tilde{t}_{1} \rightarrow t g$ receives large positive corrections that can reach roughly $50 \%$. Note that the size of the corrections is much larger than the scale uncertainty of the Born cross-section, see Fig. 4. For the next-to-leading order result, the scale uncertainty is only slightly reduced with respect to the leading-order cross-section.

\footnotetext{
${ }^{2}$ In addition, a light stop with a sizeable left-chiral component is in conflict with precision measurements of the $Z b b$ vertex.
} 

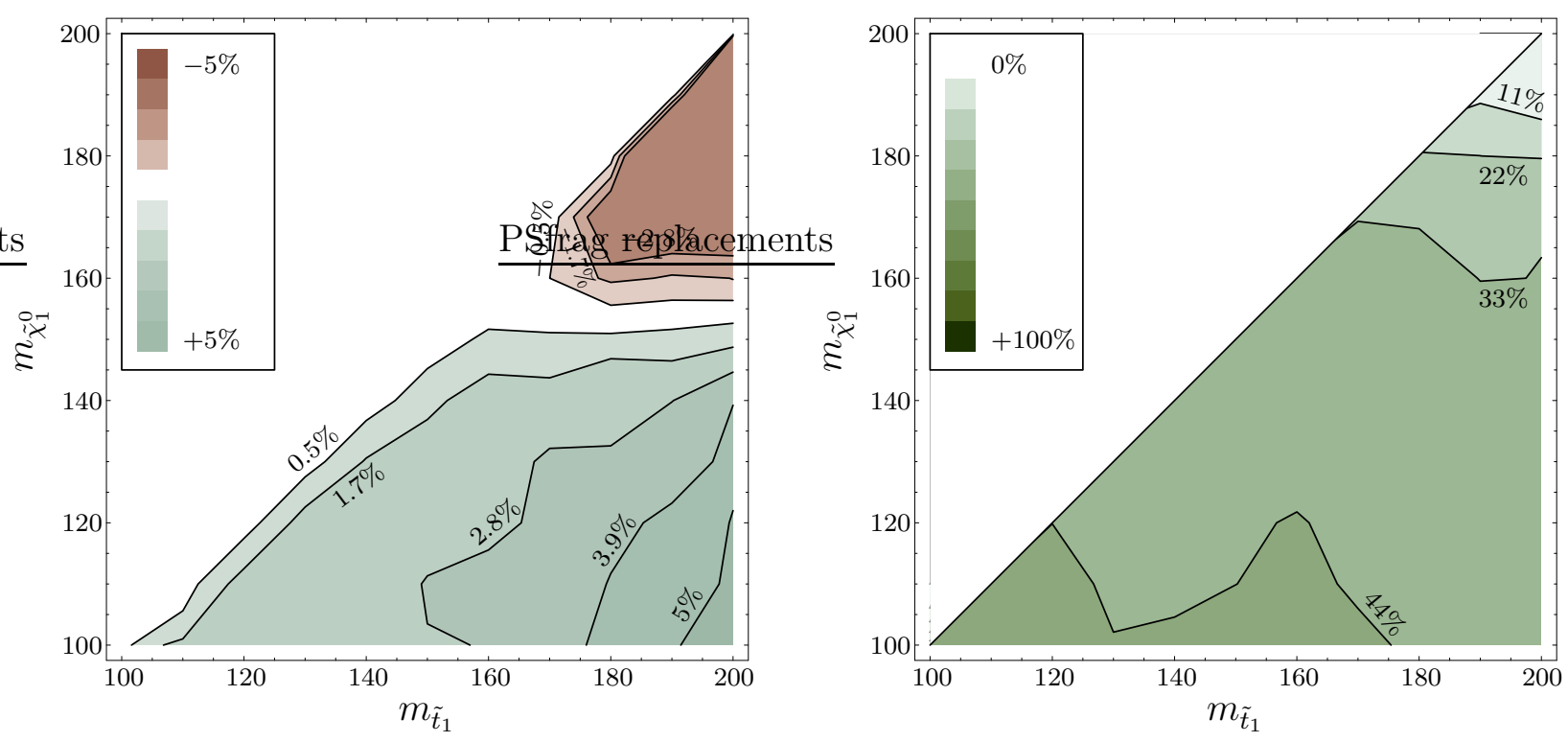

Figure 3: Relative size of the SUSY-QCD corrections to $\tilde{\chi}_{1}^{0} \tilde{t}_{1} \rightarrow W^{+} b$ (left) and $\tilde{\chi}_{1}^{0} \tilde{t}_{1} \rightarrow t g$ (right), normalized to the Born cross-sections, respectively. It is assumed that $\tilde{\chi}_{1}^{0}=\tilde{B}$ and $\tilde{t}_{1}=\tilde{t}_{\mathrm{R}}$. The other relevant supersymmetric parameters are fixed to $m_{\tilde{q}}=4$ TeV for $\tilde{q} \neq \tilde{t}_{1}$, $m_{\tilde{g}}=2 \mathrm{TeV}$, and the QCD scale is set to half the center-of-mass energy, $\mu_{0}=\sqrt{s} / 2$.

$\tilde{\boldsymbol{t}}_{\mathbf{1}} \tilde{\boldsymbol{t}}_{\mathbf{1}}^{*}$ annihilation: As mentioned above, for small stop masses $\tilde{t}_{1} \tilde{t}_{1}^{*}$ annihilation typically contributes much less to the total thermally averaged co-annihilation cross-section then $\tilde{\chi}_{1}^{0} \tilde{t}_{1}$ annihilation. Nevertheless, there are potentially very large Coulombic gluon corrections that can be important for the computation of the relic density. These corrections arise from the exchange of gluons between the stop and anti-stop. Since during the phase of freezeout, the stops and anti-stops are slowly moving $\left(E_{\text {kin } \tilde{t}} \approx T_{\text {freeze-out }} \ll m_{\tilde{t}_{1}}\right)$, long-range gluon exchange effects lead to a strong enhancement of the $\tilde{t}_{1} \tilde{t}_{1}^{*}$ annihilation cross-section. A similar enhancement effect due to QED corrections in $\tilde{\chi}_{1}^{0} \tilde{\chi}_{1}^{ \pm}$co-annihilation in the focus point region has been studied in Ref. [13].

The leading contribution shown in Fig. 5 (a) leads to a correction

$$
\sigma_{\text {coul }}=\frac{2 \alpha_{\mathrm{s}} \pi}{3 v} \sigma_{\text {Born }}
$$

where $v$ is the relative velocity between the $\tilde{t}_{1}$ and $\tilde{t}_{1}^{*}$. The divergence for $v \rightarrow 0$ is the well-known Coulomb singularity. In the case of dark matter annihilation, the Coulomb singularity is naturally cut off by the finite temperature in the early universe. Nevertheless, $v$ is typically of the same order as $\alpha_{\mathrm{s}}$, so that higher-order effects need to be included. The exchange of $n$ gluons, as in Fig. 5 (b), generates a correction factor $\propto\left(\alpha_{\mathrm{s}} / v\right)^{n}$. These effects can be systematically resummed within non-relativistic QCD (NRQCD), which is an effective theory of full QCD valid at low velocities. Within NRQCD, the problem is described by the 


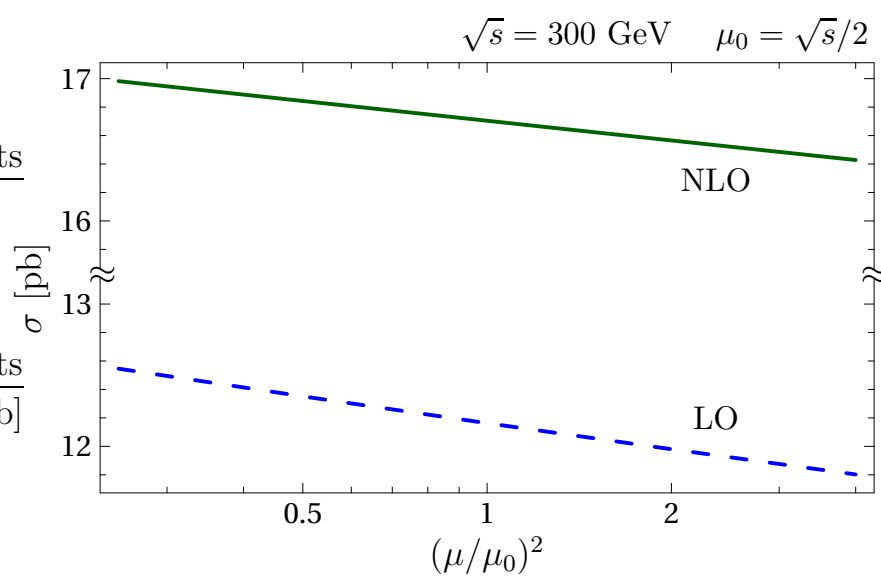

(a)

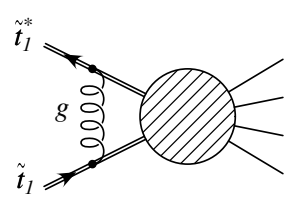

(b)

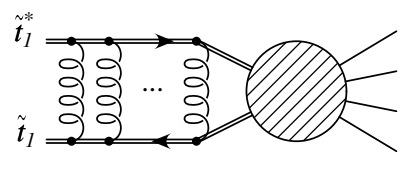

Figure 4: Dependence of the crosssection $\sigma$ for $\tilde{\chi}_{1}^{0} \tilde{t}_{1} \rightarrow t g$ on the SUSY$Q C D$ scale $\mu$ at Born (LO) and one-loop (NLO) order. The MSSM parameters are $\tilde{\chi}_{1}^{0}=\tilde{B}, \tilde{t}_{1}=\tilde{t}_{\mathrm{R}}, m_{\tilde{\chi}_{1}^{0}}=118 \mathrm{GeV}$, $m_{\tilde{t}_{1}}=138 \mathrm{GeV}, m_{\tilde{q}}=4 \mathrm{TeV}$ for $\tilde{q} \neq \tilde{t}_{1}$, $m_{\tilde{g}}=2 \mathrm{TeV}$.

Figure 5: Diagrams for the (a) leading and (b) resummed Coulombic gluon corrections to $\tilde{t}_{1} \tilde{t}_{1}^{*}$ annihilation.

Schrödinger equation with the Coulombic QCD potential $V(\boldsymbol{r})$ [14,

$$
\left[-\frac{\Delta}{m_{\tilde{t}_{1}}}+V(\boldsymbol{r})\right] \Psi(\boldsymbol{r})=(E+i \Gamma) \Psi(\boldsymbol{r}), \quad V(\boldsymbol{r})=-C_{\mathrm{F}} \frac{\alpha_{\mathrm{s}}}{r},
$$

where $C_{\mathrm{F}}$ is the Casimir operator of the $\mathrm{SU}(3)$ fundamental representation, and $E$ and $\Gamma$ are the energy and decay width of the $\left\langle\tilde{t}_{1} \tilde{t}_{1}^{*}\right\rangle$ system. The correction factor to the cross-section is then given by

$$
\frac{\sigma_{\text {coul,resum }}}{\sigma_{\text {Born }}}=|\Psi(0)|^{2} .
$$

The QCD Coulomb potential receives important higher order corrections from gluon selfinteraction and fermion loop effects [15, 16]. They can be calculated most easily in momentum space and are given by

$$
V\left(q^{2}\right)=-C_{F} \frac{4 \pi \alpha_{\mathrm{s}}}{q^{2}}\left[1+\frac{\alpha_{\mathrm{s}}}{4 \pi}\left(\frac{31}{9} C_{A}-\frac{20}{9} T_{F} n_{f}+\beta_{0} \log \left(\mu^{2} / q^{2}\right)\right)+\mathcal{O}\left(\alpha_{\mathrm{s}}^{2}\right)\right] .
$$

For a typical QCD scale choice $\mu \sim m_{\tilde{t}_{1}}$, the $\operatorname{logarithm} \log \left(\mu^{2} / q^{2}\right)=\log (1 / v)$ can become large, and it is advantageous to resum contributions of order $\mathcal{O}\left(\alpha_{\mathrm{s}} \log ^{n} v\right)$ in a similar way as the $\mathcal{O}\left(\left(\alpha_{\mathrm{s}} / v\right)^{n}\right)$ terms. This can be achieved by more elaborate effective theory frameworks, such as velocity non-relativistic QCD (vNRQCD) [17] or potential non-relativistic QCD (pNRQCD) [18. In this work, however, only a simple first estimate of the threshold corrections shall be obtained, for which the framework of NRQCD as in eq. (7) is sufficient.

The leading contribution to the $\tilde{t}_{1} \tilde{t}_{1}^{*}$ annihilation cross-section arises from the lowest S-wave state, i.e. the $\left\langle\tilde{t}_{1} \tilde{t}_{1}^{*}\right\rangle_{1 S}$ state. In eq. (7) the decay width $\Gamma$ of the stopponium state $\left\langle\tilde{t}_{1} \tilde{t}_{1}^{*}\right\rangle_{1 S}$ needs to be included. Taking into account the two largest decay channels, $\left\langle\tilde{t}_{1} \tilde{t}_{1}^{*}\right\rangle_{1 S} \rightarrow$ 


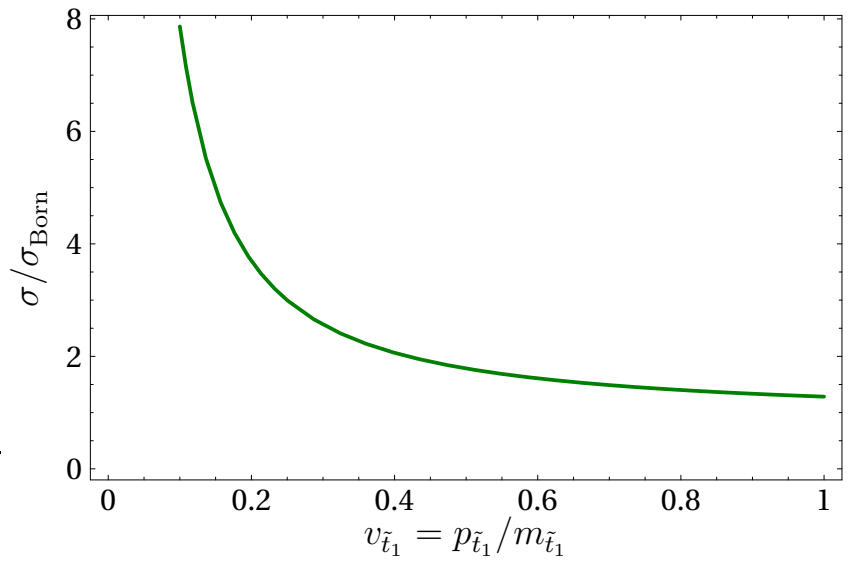

Figure 6: Cross-section for $\tilde{t}_{1} \tilde{t}_{1}^{*}$ annihilation including Coulombic QCD corrections near threshold relative to the Born cross-section, as a function of the velocity $v=\left(1-4 m_{\tilde{t}_{1}}^{2} / s\right)^{1 / 2}$. The QCD scale has been fixed to $\mu=$ $122 \mathrm{GeV}$, using five-flavor running of $\alpha_{\mathrm{s}}$ in normal QCD (not SUSY-QCD).

$g g, W^{+} W^{-}$, the partial widths at Born level read

$$
\Gamma\left[\left\langle\tilde{t}_{1} \tilde{t}_{1}^{*}\right\rangle_{1 S} \rightarrow g g\right]=\frac{448 \alpha_{\mathrm{s}}^{5} m_{\tilde{t}_{1}}}{243}, \quad \Gamma\left[\left\langle\tilde{t}_{1} \tilde{t}_{1}^{*}\right\rangle_{1 S} \rightarrow W W\right]=\frac{\alpha_{\mathrm{s}}^{3} y_{\mathrm{t}}^{4} m_{\tilde{t}_{1}}}{6 \pi^{2}}\left[1-\frac{m_{\mathrm{W}}^{2}}{m_{\tilde{t}_{1}}^{2}}+\frac{3 m_{\mathrm{W}}^{4}}{4 m_{\tilde{t}_{1}}^{4}}\right],
$$

where the second formula is valid in the limits $M_{h^{0}}^{2} \ll 4 m_{\tilde{t}_{1}}^{2} \ll M_{A^{0}}^{2}$ and $\tan \beta \gg 1$. $y_{\mathrm{t}}$ is the top Yukawa coupling. For $m_{\tilde{t}_{1}}=138 \mathrm{GeV}$, the total width is $\Gamma \approx 5 \mathrm{MeV}$, and thus almost negligible. The stop $\tilde{t}_{1}$ also has an intrinsic width, with for small mass differences $m_{\tilde{\chi}_{1}^{0}}-m_{\tilde{t}_{1}}$ is however smaller than $1 \mathrm{MeV}$ [19] and therefore can be safely ignored.

For the numerical evaluation, the Schrödinger equation with the QCD potential at twoloop order [16] is solved numerically. The result is shown in Fig. 6, which illustrates that for $v \lesssim 0.4$ the Coulombic correction can be larger than $100 \%$ of the tree-level cross-section. For very low values of $v$, the results of this calculation are not reliable, since the effect of $\tilde{t}_{1} \tilde{t}_{1}^{*}$ bounds states needs to be taken into account properly. However, as already mentioned above, the formation of bound states is inhibited by the non-zero temperature during freeze-out, so that the region $m_{\tilde{t}_{1}} v \ll T_{\text {freeze-out }}$ is not relevant for stop annihilation. In other words, in the thermally averaged integration over the annihilation cross-section, the region around $v=0$ has vanishing integration measure, so that no special treatment for the Coulomb singularity is necessary.

Effect on relic density: In order to study the effect of the radiative corrections to $\tilde{\chi}_{1}^{0} \tilde{t}_{1}$ and $\tilde{t}_{1} \tilde{t}_{1}^{*}$ annihilation, as presented in the previous sections, they have been implemented into DARKSUSY 4.1 [7]. The effect of the corrections for different neutralino and stop masses is shown in Fig. 7. In the left panel only the SUSY-QCD corrections to $\tilde{\chi}_{1}^{0} \tilde{t}_{1}$ annihilation are incorporated, which can change the predicted relic density by up to about $15 \%$. This is comparable with the current uncertainty of $\Omega_{\mathrm{CDM}}$ from astrophysical observations [1]. In the right panel, in addition the Coulombic QCD corrections to $\tilde{t}_{1} \tilde{t}_{1}^{*}$ annihilation have been taken into account. It can be seen that for small $\tilde{t}_{1}-\tilde{\chi}_{1}^{0}$ mass differences, the predicted value of $\Omega_{\mathrm{CDM}}$ can shift by more than $50 \%$ due to these effects. Even in the region which is an agreement with the current value $\Omega_{\mathrm{CDM}} h^{2}=0.1106_{-0.0075}^{+0.0056}$, the loop correction effects are larger than the current error from Ref. [1]. This is indicated by the blue band in the figure, 

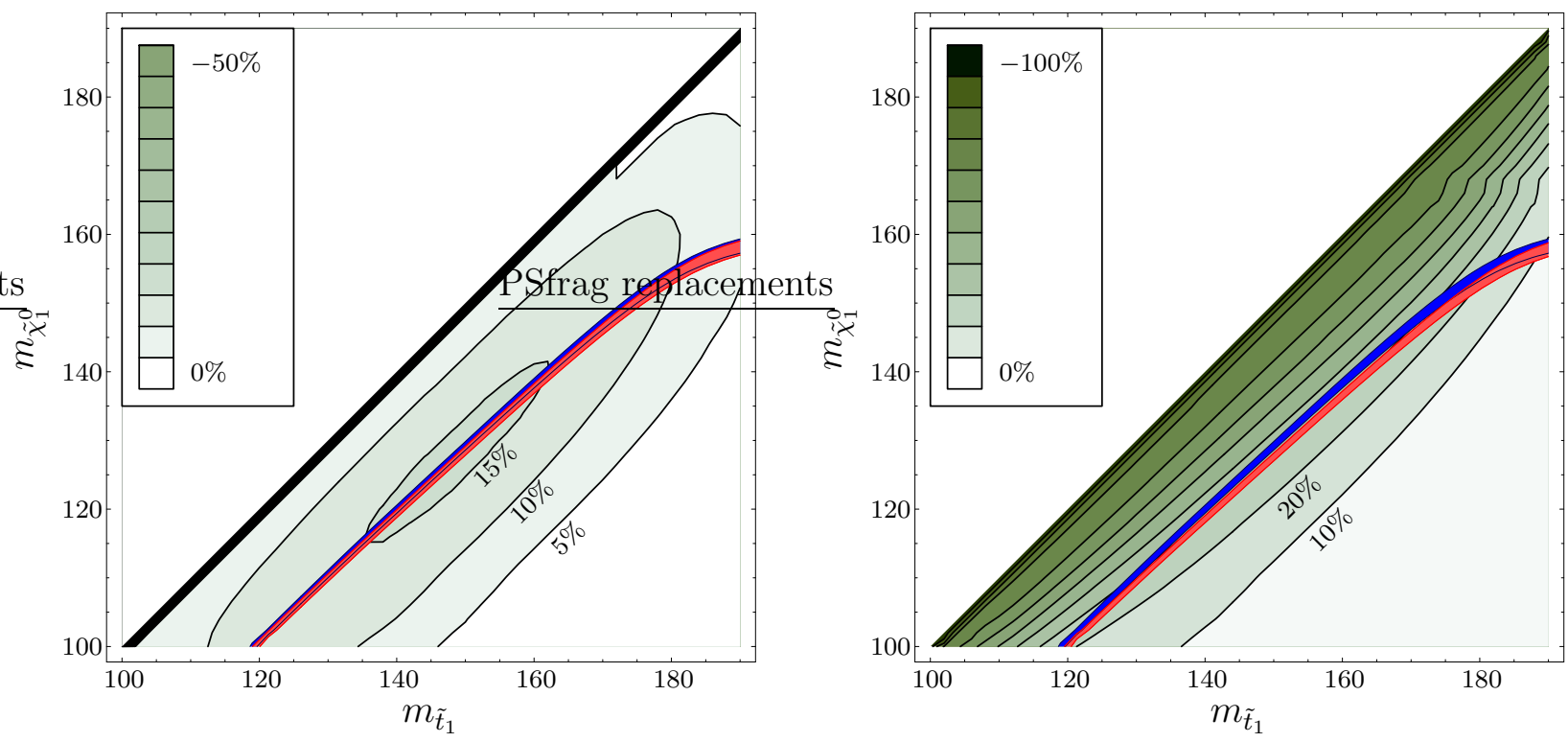

Figure 7: Relative shift of the predicted dark matter density $\Omega_{\mathrm{CDM}}$ due to radiative corrections. Left: Only SUSY-QCD corrections to $\tilde{\chi}_{1}^{0} \tilde{t}_{1}$ annihilation. Right: SUSY-QCD corrections to $\tilde{\chi}_{1}^{0} \tilde{t}_{1}$ annihilation and Coulombic $Q C D$ to $\tilde{t}_{1} \tilde{t}_{1}^{*}$ annihilation. The blue/dark and red/light bands indicate the parameter region in agreement with the observed value of $\Omega_{\mathrm{CDM}}$ without and with the radiative corrections, respectively. The parameters have been chosen as in Fig. 3.

which is the astrophysically allowed region for tree-level annihilation cross-sections, and the red band, which is the same after including the radiative corrections.

In summary, the leading $\mathcal{O}\left(\alpha_{\mathrm{s}}\right)$ corrections to neutralino-stop co-annihilation have been calculated. The process is typical for any model and scenario, where a WIMP dark matter particle annihilates together with a strongly interacting particle that can decay into the WIMP. It was found that the $\tilde{\chi}_{1}^{0} \tilde{t}_{1}$ annihilation process receives sizeable radiative corrections that modify the predicted dark matter relic density by $5-15 \%$. On the other hand, the $\tilde{t}_{1} \tilde{t}_{1}^{*}$ annihilation process receives very large corrections, that are associated with the Coulomb singularity near threshold. The effect of these Coulombic QCD corrections can have an impact on the computed relic density by more than $50 \%$ for small stop-neutralino mass differences. In a previous work by Hisano et al. [13], similarly large effects have been found as a result of QED threshold corrections in neutralino-chargino co-annihilation.

Since the leading radiative corrections presented in this paper turn out to be large, the results are expected to still have a sizeable theoretical error. For a reliable prediction of the dark matter relic density, the theoretical calculations need to be refined beyond the techniques employed in this work, including the resummation of large logarithms. However, it is interesting to note that the effect of the large QCD threshold corrections to $\tilde{t}_{1} \tilde{t}_{1}^{*}$ annihilation can also be derived from a measurement of the pair production cross-section for $e^{+} e^{-} \rightarrow \tilde{t}_{1} \tilde{t}_{1}^{*}$ at a future linear collider, see e.g. Ref. [20]. 
Acknowledgments: The author is grateful to P. Gondolo for helpful discussions and to S. Kraml and P. Gondolo for careful reading of the manuscript.

[1] D. N. Spergel et al. [WMAP Collaboration], astro-ph/0603449;

M. Tegmark et al. [SDSS Collaboration], Astrophys. J. 606, 702 (2004).

[2] M. Drees, G. Jungman, M. Kamionkowski and M. M. Nojiri, Phys. Rev. D 49, 636 (1994);

V. Barger, W. Y. Keung, H. E. Logan, G. Shaughnessy and A. Tregre, Phys. Lett. B 633, 98 (2006);

V. Barger, W. Y. Keung, H. E. Logan, G. Shaughnessy, Phys. Rev. D 74, 075005 (2006).

[3] P. Gondolo and G. Gelmini, Nucl. Phys. B 360, 145 (1991).

[4] P. Binetruy, G. Girardi and P. Salati, Nucl. Phys. B 237, 285 (1984);

K. Griest and D. Seckel, Phys. Rev. D 43, 3191 (1991).

[5] C. Balázs, M. Carena and C. E. M. Wagner, Phys. Rev. D 70, 015007 (2004);

C. Balázs, M. Carena, A. Menon, D. E. Morrissey and C. E. M. Wagner, Phys. Rev. D 71, 075002 (2005);

M. Carena, A. Finch, A. Freitas, C. Milsténe, H. Nowak and A. Sopczak, Phys. Rev. D 72, 115008 (2005).

[6] C. Boehm, A. Djouadi and M. Drees, Phys. Rev. D 62, 035012 (2000);

J. R. Ellis, K. A. Olive and Y. Santoso, Astropart. Phys. 18, 395 (2003).

[7] P. Gondolo, J. Edsjö, P. Ullio, L. Bergström, M. Schelke and E. A. Baltz, JCAP 0407, 008 (2004).

[8] T. Hahn, Comput. Phys. Commun. 140, 418 (2001).

[9] R. Mertig, M. Böhm and A. Denner, Comput. Phys. Commun. 64 (1991) 345.

[10] H. Eberl, A. Bartl and W. Majerotto, Nucl. Phys. B 472, 481 (1996);

A. Djouadi, W. Hollik and C. Jünger, Phys. Rev. D 55, 6975 (1997);

J. Guasch, J. Solà and W. Hollik, Phys. Lett. B 437, 88 (1998).

[11] W. Hollik and H. Rzehak, Eur. Phys. J. C 32, 127 (2003).

[12] G. J. van Oldenborgh and J. A. Vermaseren, Z. Phys. C 46 (1990) 425;

T. Hahn, Loop Tools 2 User's Guide (2001), [http://www.feynarts.de/looptools].

[13] J. Hisano, S. Matsumoto, M. Nagai, O. Saito and M. Senami, Phys. Lett. B 646, 34 (2007). 
[14] A. Billoire, Phys. Lett. B 92, 343 (1980);

Y. Sumino, K. Fujii, K. Hagiwara, H. Murayama and C. K. Ng, Phys. Rev. D 47, 56 (1993).

[15] L. Susskind, in Weak and electromagnetic interactions at high energy, eds. R. Balian and C. H. Llewellyn-Smith, North Holland, Amsterdam (1977);

W. Fischler, Nucl. Phys. B 129, 157 (1977).

[16] Y. Schröder, Phys. Lett. B 447, 321 (1999).

[17] M. E. Luke, A. V. Manohar and I. Z. Rothstein, Phys. Rev. D 61, 074025 (2000);

A. V. Manohar and I. W. Stewart, Phys. Rev. D 62, 074015 (2000);

A. H. Hoang and I. W. Stewart, Phys. Rev. D 67, 114020 (2003);

A. H. Hoang and P. Ruiz-Femenía, Phys. Rev. D 73, 014015 (2006).

[18] N. Brambilla, A. Piñeda, J. Soto and A. Vairo, Nucl. Phys. B 566, 275 (2000);

A. Piñeda and A. Signer, Nucl. Phys. B 762, 67 (2007).

[19] K. i. Hikasa and M. Kobayashi, Phys. Rev. D 36, 724 (1987).

[20] A. Freitas, C. Milsténe, M. Schmitt, A. Sopczak, in preparation. 